\title{
ESPACIO RELACIONAL, PROCESO INNOVADOR Y DIFUSIÓN TERRITORIAL DE LA INNOVACIÓN EN ANDALUCÍA*
}

\author{
F. Ruiz Rodríguez \\ Departamento de Geografia Física y Análisis Geográfico Regional. Universidad de Sevilla. \\ C/ María de Padilla, s/n C.P. 41004. Sevilla. \\ fruiz@us.es
}

\begin{abstract}
Resumen: Este trabajo analiza las relaciones de conocimiento que establecen las empresas andaluzas con otros agentes del entorno productivo local, regional, nacional, europeo y resto del mundo para el desarrollo de actividades de I+D. A partir de la información que suministra 156 cuestionarios-entrevistas se estudia sus características espaciales, los factores o causas que las definen; y los efectos tanto en el proceso innovador de las propias firmas como el papel que juegan en la difusión territorial de la innovación en la Comunidad Autónoma. Como suele ocurrir en las regiones periféricas, la malla de flujos entre el subsistema empresarial y el científico, administrativo, etc, es de reducida complejidad tecnológica. Los procesos de difusión de la innovación a escala regional y local emanan de las empresas de servicios avanzados y las manufacturas de media tecnología, y desde un punto de vista territorial de las grandes áreas urbanas de la región.
\end{abstract}

Palabras clave: Investigación y Desarrollo Tecnológico (I+D), Innovación, Proceso Innovador, Sector empresas, Difusión Territorial, Regiones periféricas, Redes, Andalucía.

\begin{abstract}
This issue analyses the knowledge relationships which Andalusian firms establish with other agents from the local, regional, national and European environment and the rest of the world for the development of Research and Development $(\mathrm{R}+\mathrm{D})$ activities. From the information provided by 156 questionnaires-interviews it is studied their spatial characteristics the factors or causes which define them; and the effects in the innovating process of the firms themselves as well as their role on the territorial diffusion of innovation within the Autonomous Community. As it may happen in peripheral regions, the network among the managerial subsystem and the scientific subsystem, the administrative one, etc., is of a reduced technological complexity. The diffusion process of
\end{abstract}

* Recibido: 09-12-04. Aceptado: 16-06-05. 
innovation at regional and local levels emanates fron the advanced services firms and fron medium techology manufactures, and from a territorial point of view, from the great urban areas in the region.

Key words: Technological Research and Development $(R+D)$, Innovation, Innovation process, Business Sector, Territorial Distribution, Peripheral Regions, networks, Andalusian.

\section{Introducción}

En nuestros días los contrastes espaciales a cualquier escala de estudio (regional, intrarregional) tienen más bien caracteres cualitativos que cuantitativos (Méndez, 1997a) ya que son el resultado de las diferentes capacidades que gozan los territorios para producir o incorporar innovaciones. La situación relativa que ocupan las regiones, en función de su nivel de desarrollo, en el marco europeo o a cualquier otra escala de estudio, está asociada claramente a la capacidad innovadora de su tejido productivo. Y especialmente, a la tenencia de un fuerte subsistema empresarial de innovación que traduzca en productos nuevos o mejorados en el mercado los nuevos conocimientos (tecnología) producidos o adquiridos por el sistema de innovación regional.

Desde la disciplina geográfica, y para las regiones periféricas, es de suma importancia conocer y verificar como el espacio relacional de carácter productivo y cognoscitivo organiza, dinamiza, al territorio regional y se convierte en palanca de la economía, desarrollando factores específicos de carácter endógeno (Jordá, 2003 a). A este tipo de regiones le interesa que la creación de $\mathrm{I}+\mathrm{D}$ e innovación se difunda por todo el territorio y no se externalice en parte fuera de ésta al no encontrar la suficiente demanda en el ámbito territorial. Es necesario tener presente que la creación y absorción de conocimientos en las empresas no depende sólo de los esfuerzos individuales de cada uno de ellas sino también de toda aquella información a la que se tiene acceso. Por ello es la eficacia de la organización de todo el conjunto de empresas e instituciones localizadas en un lugar o en un área la que explica el éxito del proceso innovador de un territorio (Jordá, 2003 b).

Las dificultades que tiene el tejido productivo andaluz para innovar y realizar actividades de $\mathrm{I}+\mathrm{D}$, situándose en términos relativos y en relación con otras regiones europeas en los puestos más bajos ya sea por el gasto interno total en $\mathrm{I}+\mathrm{D}$ como en otros indicadores, parece estar asociado a la insuficiente capacidad del sector empresarial para absorber la innovación resultante de las actividades de $\mathrm{I}+\mathrm{D}$ regionales (Ruiz, 2003). No se debe olvidar que una de las muchas debilidades del desarrollo 
andaluz, y desde una óptica macroeconómica, es la deficiente articulación productiva que provoca efectos limitativos en el crecimiento global y sectorial interno, y que origina una fuerte dependencia de la región del exterior. El proceso de globalización de la economía y las características estructurales de las empresas españolas indican que España y en particular Andalucía están enfrentadas a una grave crisis de competitividad. Crisis que se identifica, entre otros aspectos, con la insuficiencia tecnológica, el tamaño empresarial, fundamentalmente PYMES, y la escasa cooperación entre ellas (Vallés, 1997 a y b).

La Comunidad Autónoma andaluza posee un sistema de innovación regional característico de las regiones periféricas ${ }^{1}$, en las que predominan las Universidades como principal ejecutor de las actividades de I+D (Ruiz, 2003). Como señala Jordá, R. (1997) en Andalucía el entorno científico es el único que reúne condiciones para fomentar la cooperación con el subsistema productivo y tecnológico dado que ha alcanzado la madurez en la tasa de doctores por cada 1000 habitantes sobrepasando la media española, y en el peso conseguido por cada área prioritaria (20\%) en el gasto de $\mathrm{I}+\mathrm{D}$ nacional al superar la importancia que tiene la región en población y en VAB respecto al conjunto español. A finales del año 2003 sólo un poco más de 2000 empresas-establecimientos andaluzas realizan algún tipo de innovación (proceso/producto, interna/mercado), y únicamente unas 300 llevaban a cabo actividades de I+D como mecanismo principal o secundario para acometer innovaciones de carácter tecnológico u organizativo, según los resultados obtenidos por trabajos realizados en el seno del grupo de investigación Estudios Geográficos Andaluces.

\section{Objetivos y Metodología}

La investigación parte del conocimiento de que las regiones menos desarrolladas tienen grandes dificultades para poseer un sistema de innovación regional integrado tanto desde el punto de vista espacial como en sus agentes (públicos y privados). En este contexto, el objetivo principal de este trabajo ha sido: a) el estudio de las características de las relaciones/redes de conocimiento que establecen las empresas-establecimientos que realizan actividades de $\mathrm{I}+\mathrm{D}$, los factores o causas que las definen; $y$ b) los efectos de éstas tanto para el proceso innovador de las propias firmas de $\mathrm{I}+\mathrm{D}$ como el papel que juegan en la difusión territorial de la innovación en la Comunidad Autónoma.

Este estudio no sólo pretende aumentar el conocimiento de esta realidad, sino también aplicar una metodología en donde esté presente la variable espacial. Para ello, y ante la inexistencia de información estadística y de otro tipo sobre el objeto de estudio ${ }^{2}$, la investigación se fundamenta en: a) la explotación de la base de datos 
de empresas-establecimientos innovadoras y de I+D de Andalucía ${ }^{3}$ y b) el análisis de la información que nos suministra 156 encuestas-entrevistas ${ }^{4}$ a empresas-establecimientos que realizan actividades de I+D en Andalucía. Las entrevistas que se llevaron a cabo a lo largo del año 2001 por quien suscribe este trabajo se basan en un cuestionario de unas 600 variables que cubren los campos que vamos a desarrollar en este artículo.

Para alcanzar este propósito, el enfoque adoptado ha sido el sistémico, en particular se sostiene en el concepto de sistema nacional o regional de innovación que adjudica una gran importancia a la proximidad espacial, las externalidades, la cultura e identidad regional y el proceso de aprendizaje colectivo o regional en la creación y difusión de las innovaciones (Koschatzky, 2000). Se entiende por Sistema de Innovación Nacional y/o Regional ${ }^{5}$ los sistemas compuestos por las organizaciones e instituciones de un país que influyen en el desarrollo, difusión y uso de las innovaciones (Edquist, 1997: 14). De esta forma, innovar a través de actividades de $I+D^{6} \mathrm{o}$ de otras actividades innovadoras, se considera un proceso complejo e iterativo en el que el conocimiento tácito, las competencias específicas, los activos complementarios (Teece, 1987) y los sistemas nacionales de innovación desarrollados (Lundvall, 1992) juegan papeles decisivos. La innovación necesita por tanto de la adquisición y síntesis de muchos tipos de conocimientos, y el propio conocimiento debe ser transformado una vez adquirido de fuentes externas, que pueden estar en el mismo territorio o fuera de éste. El conocimiento fluye a través de redes, cada vez más complejas, que conllevan dinámicas tanto de colaboración como de competencia.

Por otra, se apoya en la teoría porteriana del Desarrollo Competitivo de las Naciones ya que se cimienta en la idea de que si las empresas innovan para ser competitivas en sus mercados o para ampliar éstos últimos, un territorio es más competitivo cuanto mayormente posea una red de empresas relacionadas de forma vertical (cliente-proveedor) y horizontal (intra e intersectorial) en productos especializados o innovadores (Porter, 1990). En ella se defiende que el proceso de "perfeccionamiento" de la economía de un espacio (nación, región...), y el alcance del desarrollo que se adquiere por éstos requiere que las empresas del lugar, únicos agentes verdaderos del desarrollo competitivo de las "naciones", mejoren continuamente su tecnología y su capacidad de innovación. Además, se insiste en que la actuación de los poderes públicos juega un gran papel en la dinamización de los territorios en tanto pueden incitar e incluso presionar a las empresas para que mejoren. Igualmente reafirma las consideraciones del desarrollo del territorio desde el punto de vista local.

Para la teoría del desarrollo endógeno, como señala Aydalot (1986 a y b), el desarrollo económico de un territorio no se apoya sólo en la capacidad de adquirir tecnología sino que depende a su vez de la capacidad innovadora del sistema productivo de cada localidad. La magnitud de la innovación en un territorio viene dada por la confluencia de los intercambios de productos y recursos entre las empresas, la mul- 
tiplicidad de las relaciones entre los actores, la transmisión de mensajes e informaciones que existan entre ellos y la implicación de la administración pública en el desarrollo innovador y global de algunas localidades y regiones. Estas relaciones propician la difusión de innovaciones, impulsan el aumento de la productividad y mejoran la competitividad de las empresas locales (Vázquez, 1997).

Por último, y también, sigue a la teorías de la innovación, la del cambio técnico inducido y el modelo teórico propuesto por Dematteis (1995) por el que la dinámica del territorio, a través de formas de organización de la producción y del conocimiento, viene condicionada por cuestiones sectoriales, es decir con el tipo de actividades presentes (sectores innovadores o maduros, en expansión o en regresión ${ }^{7}$ ), con la estructura de las empresas que en ellos operan (pequeñas o grandes y sus enlaces), con la gama de especializaciones presentes y a la historia particular de cada nodo, comarca o región como queda patente en el trabajo de Salom et al. (1999) para la Comunidad Valenciana.

En este marco, la investigación centra su atención por una parte en la propensión a cooperar y/o colaborar de estas empresas para innovar, y por otra en el espacio relacional que se establece con los diferentes agentes que intervienen en su proceso innovador en función del sector tecnológico al que pertenece y la localización de las organizaciones/instituciones implicadas en las redes. Para ello se consideran dos tipos de relaciones:

a) Las vinculadas directamente con la realización de actividades de I+D: cooperación en proyectos de $\mathrm{I}+\mathrm{D}$ con otras empresas (subsistema empresarial) y con centros de investigación públicos (OPIs)- Universidades (subsistema científico), la compra de tecnología inmaterial y las relaciones con la Administración a través del apoyo a proyectos de $\mathrm{I}+\mathrm{D}$.

b) Las insertas en actividades de producción que indirectamente se pueden convertir en fuente de conocimiento para el desarrollo de actividades de I+D: relaciones proveedor-cliente, adquisición de maquinaria y equipo mecánico, subcontratación con proveedores- clientes y lo vínculos con las empresas de servicios avanzados.

Para el análisis sectorial de los datos se parte de la Clasificación Nacional de Actividades Económicas (CNAE-93), y el estudio agregado y espacial de la especialización tecnológica del territorio se ha llevado a cabo a partir de dos clasificaciones:

a) La Taxonomía de Pavitt (1984) para las manufacturas, y la de Soete y Miozzo (1989) para las actividades de servicios. A partir de ellas podemos acercarnos a los patrones de innovación de las empresas-establecimientos catalogando la industria en cuatro categorías: Dominadas por los proveedores (DP), produc- 
toras a gran escala (PGE), proveedoras especializados (PE) y de base científica (BC). Las firmas de servicios se pueden reagrupar en sectores dominados por los proveedores, servicios intensivos en producción, intensivos en escala y servicios de redes, proveedores especializados en tecnología y sectores de base científica.

b) La clasificación sectorial según intensidad tecnológica de la OCDE 1997, basada en la función gastos en $\mathrm{I}+\mathrm{D}$ /producción de las ramas CNAE. Ésta nos permite agregar las empresas-establecimientos en cuatro sectores según su grado tecnológico: Alta, Media-Alta, Media y Baja Tecnología.

Este procedimiento tiene ciertas limitaciones, de carácter general, derivadas de las características de las dos clasificaciones, y en particular de su aplicación a regiones con bajo nivel de desarrollo, como es el caso de Andalucía. Entre ellas podemos destacar:

a) Ambas resaltan mucho la innovación tecnológica, olvidándose de la no tecnológica, tan frecuente en el sector servicios.

b) La existencia de sectores como el de las comunicaciones o empresas de servicios avanzados que pueden situarse en más de una categoría.

c) La asignación de los sectores CNAE a los grupos de Pavitt no es fácil, y sería necesario para una adscripción más correcta utilizar una desagregación de la CNAE a 4 dígitos. Y cuando se refleja en un mapa la localización de algunos de los sectores económicos de base científica y de proveedores especializados, la información suele ocultar la presencia simultánea de grandes y pequeñas firmas con capacidad tecnológica diferente, así como establecimientos donde puede realizarse tareas de valor y contenido innovador muy desigual, asociados a puestos de trabajo con niveles de cualificación contrastados (Méndez, R., 1997 b).

d) Al igual que la anterior, también la clasificación de la OCDE encuentra grandes dificultades de aplicación por los continuos cambios que sufren los listados en función del ciclo de vida de las tecnologías. Y en concreto, su aplicación en regiones atrasadas ya que son muy grandes las diferencias existentes entre éstas con las de los países en donde la OCDE realizó sus cálculos.

Mientras que para el análisis espacial se utiliza: a) las cuatro escalas más usadas en las estadísticas (local, regional, nacional, europeo y resto del mundo) para referenciar los flujos); y b) la comarcal en la localización de las empresas de I+D. La base comarcal adoptada ha sido la propuesta por el Grupo de Investigación Estudios Geográficos Andaluces bajo la dirección del Dr. Cano García (Cano, 2002) (Anexo I) 
puesto que su elaboración ha tenido en cuenta múltiples criterios, principalmente funcionales, y otros como la agrupación voluntaria de municipios en mancomunidades, comarcas de desarrollo y la atención a las áreas periféricas desarticuladas.

\section{El espacio relacional de la cooperación}

La tendencia a cooperar de las empresas que realizan actividades de $\mathrm{I}+\mathrm{D}$ en la región es relativamente alta $(64,20 \%$ de las empresas) (Figura 1). Pero sólo el 44,6\% y el $29,6 \%$ de las firmas se asocian con centros públicos de investigación-universidades y otras empresas respectivamente para desarrollar su tecnología (cuadro 1). Durante el periodo comprendido entre 1996-2001 se han llevado a cabo un reducido número de proyectos, tan sólo uno para la mayor parte de las empresas encuestadas (75\% de las que colaboran con el subsistema científico y el $66,5 \%$ para las que cooperan con otras empresas).

La poca disposición a cooperar de las empresas está asociada, una vez más, a las características estructurales e innovadoras de las ramas o sectores productivos a los que pertenecen. Por una parte, existe una mayor tendencia a cooperar de las empresas industriales con centros públicos, y desciende a medida que aumenta la intensidad tecnológica del sector al que pertenece, sobre todo en aquellas cuyos procesos de innovación vienen definidos por los proveedores especializados. Mientras que los servicios avanzados recurren mucho menos a la colaboración y cuando lo hacen se establece con otras empresas (cuadro 1).

El circuito formal de la red de relaciones externas en la práctica de la I+D está reservado, y según los resultados de las encuestas, a las empresas más dinámicas, pequeñas o grandes, y que están en mejor posición competitiva regional, nacional e internacional. El suficiente nivel de experiencia técnica y comercial, conocimientos y madurez como para ser capaz de detectar oportunidades de innovación a través de la cooperación se encuentra en: a. las grandes firmas de baja tecnología dominadas por los proveedores y productores a gran escala participadas por capital nacional y b. en un reducido número de empresas de media-alta y alta tecnología.

Por el contrario a como cabria esperar las empresas medianas (1,50 a 3 millones de euros de facturación) de media-alta y alta tecnología tienen una escasa propensión a cooperar en proyectos de I+D (cuadro 2). Según las investigaciones realizadas por Jordá, R. et al. (2000) este hecho se debe a que es muy reducida la complejidad de sus procesos de innovación y por lo tanto de los productos resultantes. En este caso predominan relativamente las relaciones de subcontratación con grandes multinacionales que no poseen en la región los centros de I+D . 
Cuadro 1. Cooperación del Subsistema Empresarial de $1+D$ en los últimos 6 años (\% del número de empresas).

\begin{tabular}{|lcc|}
\hline Clasificación sectorial & Universidad-OPIs & Empresas \\
\hline INTENSIDAD TECNOLÓGICA & & \\
\hline Primario Baja Tecnología (PBT) & 100 & 66,67 \\
Manufacturas Baja Tecnología (MBT) & 66,7 & 33,3 \\
Manufacturas de Media Tecnología (MMT) & 57,6 & 31,9 \\
Manufacturas Media-alta y Alta Tecnología (MMAT) & 40,5 & 32,7 \\
Servicios de Baja Tecnología (SBT) & 100,0 & 0,0 \\
Servicios de Media Tecnología (SMT) & 32,4 & 30,7 \\
Servicios de Alta Tecnología (SAT) & 0,0 & 16,7 \\
TOTAL & 44,6 & 29,6 \\
\hline PATRONES DE INNOVACIÓN & & 66,67 \\
\hline Primario dominado por Proveedores (PDP) & 100 & 16,7 \\
Manufacturas dominadas por los Proveedores (MDP) & 83,3 & 37,8 \\
Manufacturas de Producción a Gran Escala (MPGE) & 53,3 & 19,6 \\
Manufacturas de Proveedores Especializados (MPE) & 51,8 & 50,0 \\
Manufacturas de Base Científica (MBC) & 16,7 & 0,0 \\
Servicios de Producción a Gran Escala (SPGE) & 100,0 & 24,7 \\
Servicios de Base Científica y de Proveedores Especializados (SBC/SPE) & 18,5 & 29,6 \\
TOTAL & 44,6 & \\
\hline
\end{tabular}

Elaboración propia a partir de las encuestas.

Cuadro 2. Proyectos de I+D desarrollados con centros públicos (OPIs y Universidades) y con empresas por tamaño empresarial (\% del número de empresas).

\begin{tabular}{|lcc|}
\hline Tamaño * & Universidad-OPIs & Empresas \\
\hline Menos de 0, 60 & 20,58 & 30 \\
0,60 a 1,50 & 22,05 & 17,5 \\
1,50 a 3 & 5,88 & 10 \\
3 a 30 & 22,05 & 22,5 \\
Más de 30 & 29,41 & 20 \\
Total & 100 & 100 \\
\hline
\end{tabular}

Elaboración propia a partir de las encuestas.

* Tamaño según facturación (millones de euros)

Por otra, se definen unas redes colaboración en proyectos de $\mathrm{I}+\mathrm{D}$ muy simples ( $80 \%$ de ellas), muy elementales, formadas por dos empresas o una empresa y un centro de investigación en las que predomina la componente local-regional con los centros públicos de investigación, y nacional-extranjero en las relaciones de colaboración interempresariales (cuadro 3). Sólo el 23,3\% de las empresas han formalizado, al amparo de políticas públicas, redes de cooperación más complejas en las que se insertan empresas y centros públicos de investigación (20\% de las redes). Así podemos diferenciar desde el punto de vista de la dimensión espacial: 
Cuadro 3. Tipología espacial de las Redes de Cooperación con Opis-Universidad y Empresas (\% de redes).

\begin{tabular}{|l|l|c|c|c|c|c|c|}
\hline & CENTRO & \multicolumn{5}{|c|}{ EMPRESA } \\
\hline SIMPLE & & LOC $^{*}$ & REG & NAC & \multicolumn{2}{|c|}{ EXT } & TOTAL \\
\hline & LOC & 8 & 12 & 12 & 16 & 48 \\
& REG & & & 8 & 16 & 24 \\
& NAC & & & 8 & & & 8 \\
& EXT & & & & & & 20 \\
& TOTAL & LOC-REG & & & LOC-EXT & EXT & \\
\hline & & 4 & & & & & 4 \\
\hline COMPLEJA & LOC-REG & & & & 4 & & 4 \\
& LOC & & & & & 4 & 4 \\
& REG-NACIONAL & & & & & 4 & 4 \\
& LOC- NAC & & & & & 4 & 4 \\
& REG-NAC-EUR & & & & & & 20 \\
\hline
\end{tabular}

Elaboración propia a partir de las encuestas.

* LOC: local.; REG: regional; NAC; nacional;EXT: extranjero; LOC-REG: local-regional: LOC-EXT: local-extranjero; EUR: europeo.

1. Una elevada concentración local de los flujos en las relaciones con el subsistema científico. Entre el $60-70 \%$ de los contratos establecidos por las empresas encuestadas se realiza con centros públicos-universidades de la provincia en la que está ubicada. Prácticamente la mitad de las redes abarca geográficamente el ámbito local, sobre todo las que se desarrollan a partir de las firmas ubicadas en el área metropolitana de Sevilla. Únicamente el 29\% de las relaciones tienen un alcance nacional, en donde el centro cooperante se sitúa principalmente en Madrid (58\%), y les siguen, a gran distancia, los de Barcelona, ciudades del País Vasco, Valencia, Salamanca y Valladolid.

En el $74,6 \%$ de las redes de cooperación en proyectos de I+D está presente el subsistema científico regional, generalmente la Universidad; mientras que los centros nacionales y europeos sólo forman parte del 22,03\% y el 3,39\% respectivamente de las redes. En especial, las empresas situadas en el área metropolitana de Sevilla acogen el 51,5\% del total de convenios firmados con centros públicos en la región, y sólo en el 27,28\% de ellos participan empresas de otras provincias andaluzas, destacando las malagueñas $(33,3 \%)$ y las onubenses (25\%). Del resto de capitales provinciales las que más atraen son Córdoba y Almería (25\% y $20 \%$ de las interprovinciales respectivamente).

Esto se debe de acuerdo con la información derivada de los cuestionarios a la conjunción de cuatro factores:

1. La inexperiencia de las empresas en cuestiones de colaboración.

2. La necesidad de contactos cara a cara para discutir y resolver problemas.

3. A la reducida capacidad para desarrollar proyectos competitivos de alto nivel y acceder a programas nacionales, europeos e internacionales de $\mathrm{I}+\mathrm{D}$.

4. La especialización en tecnologías en Ciencias de la Vida de la I+D pública. 


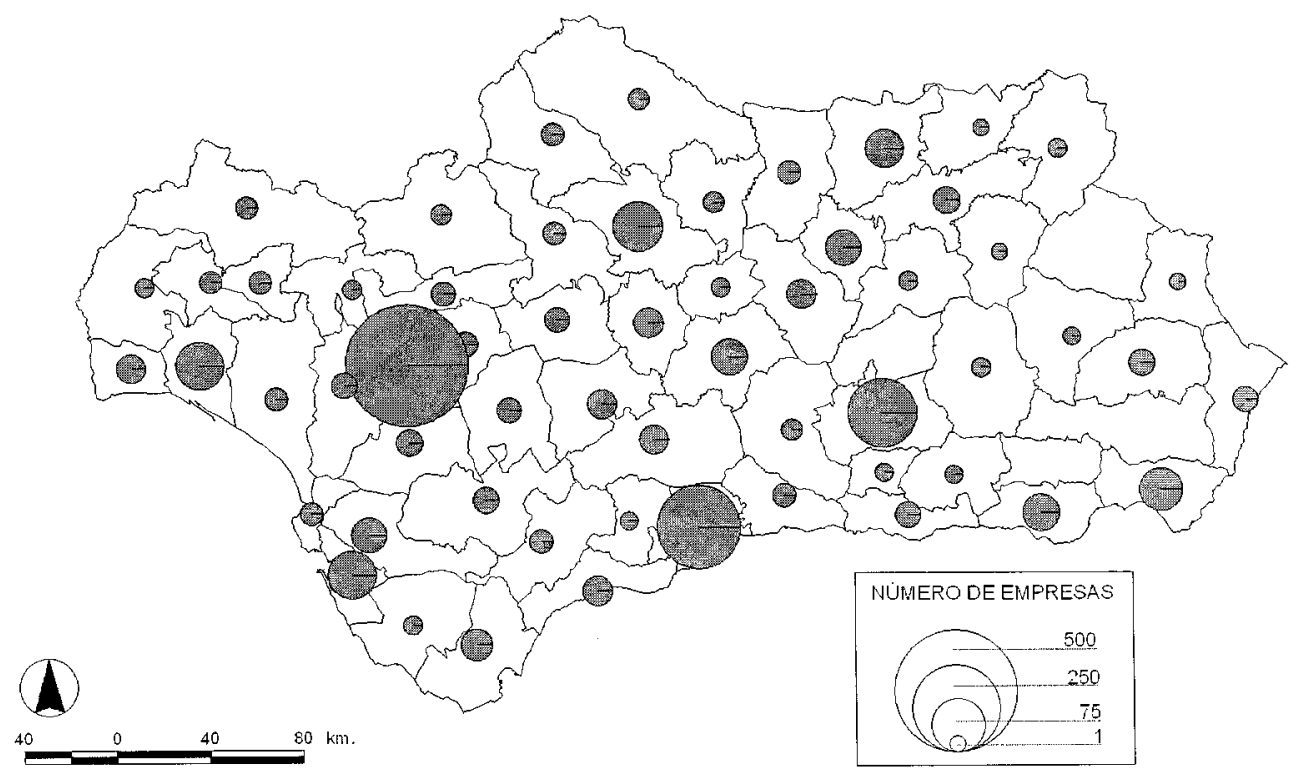

Mapa 1. Localización comarcal de las empresas innovadoras. Fuente: Elaboración propia.

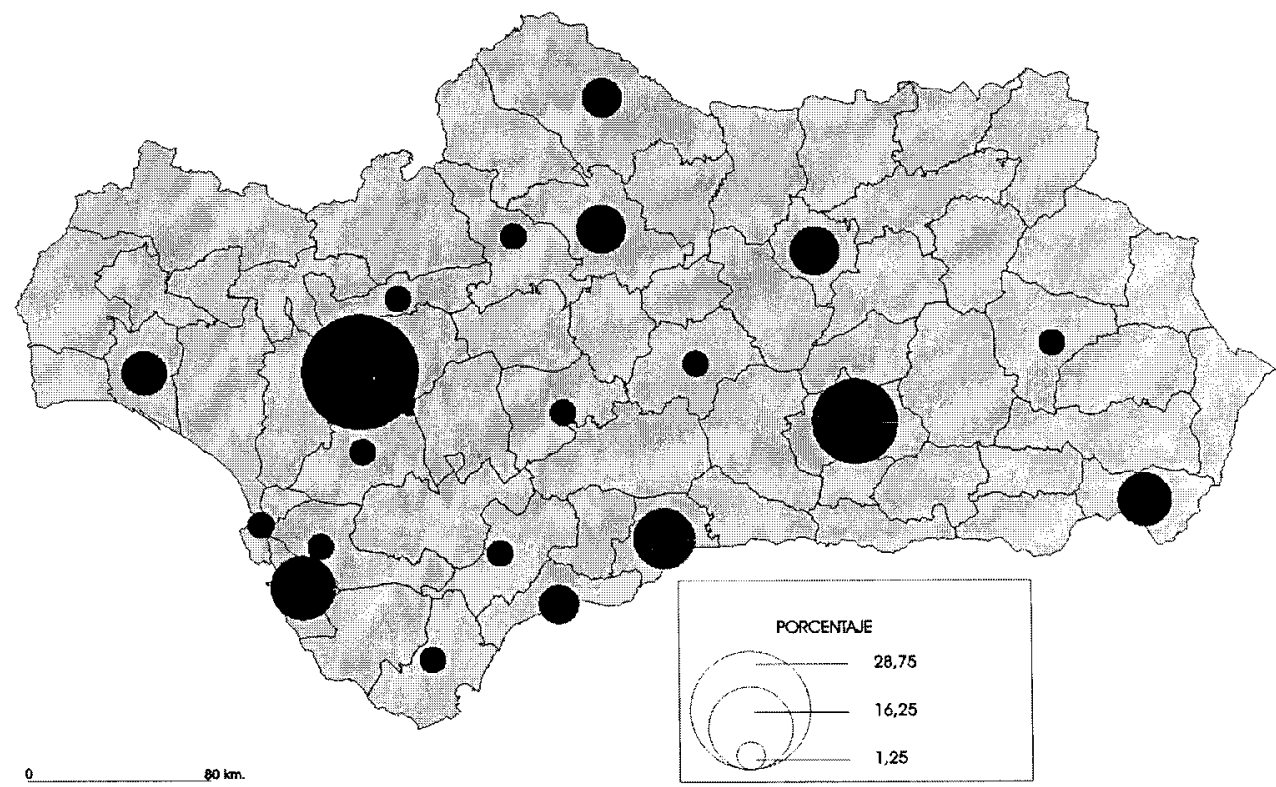

Mapa 2. Localización comarcal de los centros-organismos públicos de I+D.

Fuente: Elaboración propia a partir de la base de datos de Centros y Organismos Públicos de 1+D de Andalucía. 
La escasa preparación y el bajo nivel tecnológico del subsistema empresarial de $\mathrm{I}+\mathrm{D}$, fundado con la inversión en la investigación aplicada y desarrollo tecnológico para la mejora de productos, hace que sólo se coopere en I+D para desarrollar tecnologías ya existentes, adaptaciones o estudios sobre las potencialidades de éstas, sobre todo en campos científicos ligados a Ciencias de la Vida en función de la demanda productiva regional; y con centros muy próximos geográficamente, lo cual se ve favorecido por la elevada concentración territorial de la innovación en Andalucía (mapa 1 y 2 ).

2. Las relaciones formales entre empresas para el desarrollo de actividades de I+D tienen igualmente un carácter regional (40,74\%) (Figura 1). Si bien el 31,4\% de las redes que se establecen poseen una dimensión espacial foránea, en las que participan empresas de otras comunidades autónomas españolas (31\%), de la Unión europea $(16,6 \%)$ y extranjeras $(11 \%)$.

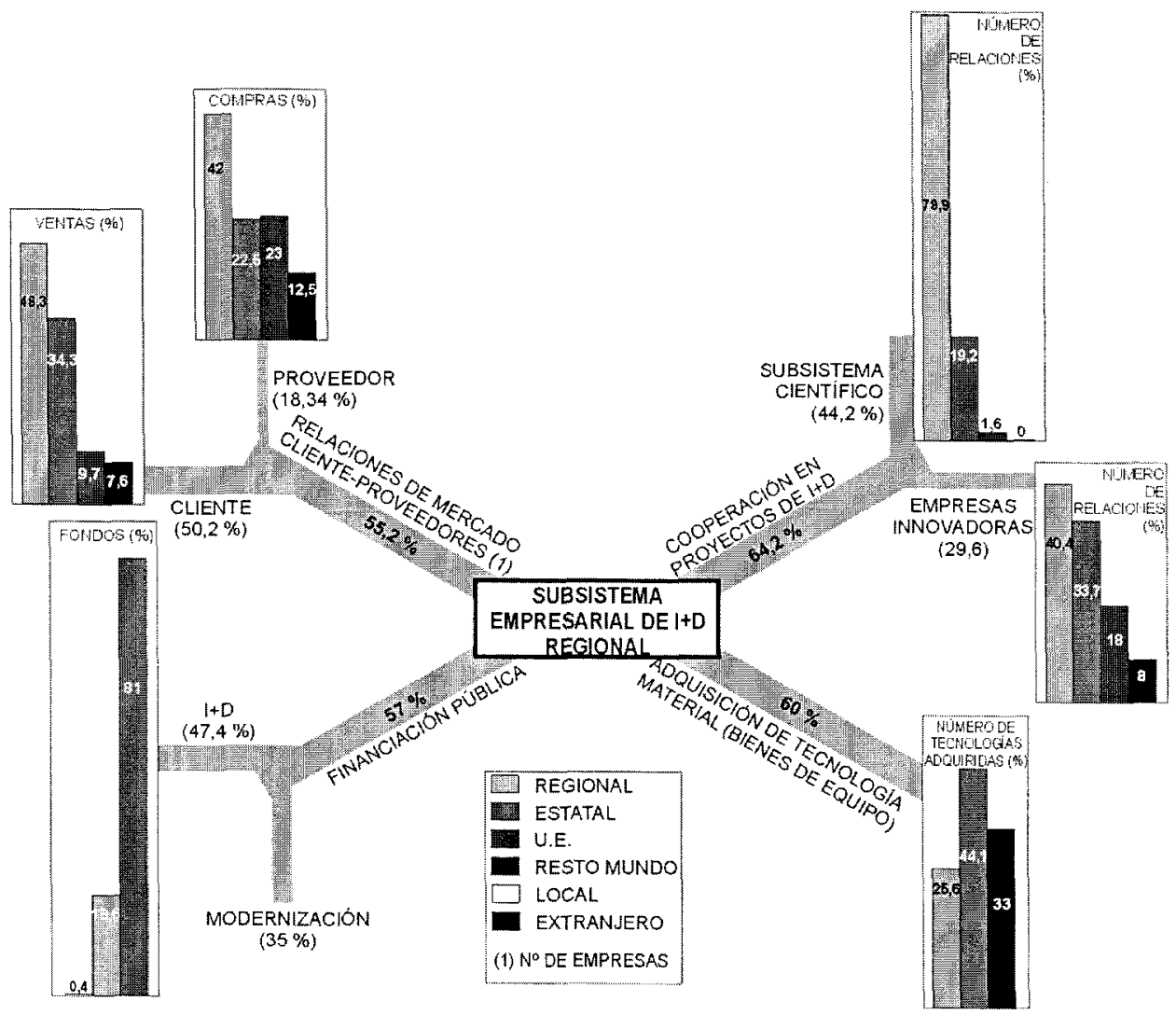

Figura 1. Principales fuentes de conocimiento para la I+D empresarial. Fuente: Elaboración propia a partir de las encuestas. 
Al contrario de lo que ocurría en las relaciones con el subsistema científico, la cooperación con otras empresas está relacionada más claramente con el contenido tecnológico de los sectores a los que pertenecen. En primer lugar, los servicios avanzados de media y alta tecnología y de base científica (consultoría e ingeniería) acaparan el $50 \%$ de los proyectos de I+D interempresariales. Se realizan con: a. proveedores del propio sector (empresas o autónomos), del resto de servicios y manufacturas de alta tecnología, y b. otras ramas del tejido productivo a petición de los clientes. Le siguen las manufacturas de media y media-alta y alta tecnología de base científica, productores a gran escala e incluso las dominadas por proveedores (cuadro 4).

El análisis sectorial de las respuestas de la encuesta nos muestra como el ámbito europeo es importante para las de media tecnología, y el nacional para las de media-alta y alta tecnología. Éstas últimas (electrónico, químico, etc.) cooperan fundamentalmente con otras empresas industriales y de servicios del mismo grado tecnológico. Mientras que las de media tecnología (agroalimentario, minerales no motálicos, etc.) lo hacen tanto con las de baja tecnología, con ellas mismas como con las altas tecnologías (cuadro 5).

En ambos casos cooperan fundamentalmente con empresas proveedoras de equipo y de insumos especializados de producción y de servicios avanzados, aunque tienen cierta importancia los clientes. Se trata de ramas con una fuerte presencia de PYMEs, especialmente firmas de tamaño mediano proveedoras de inputs a grandes clientes de capital nacional o extranjero que mantienen relaciones comerciales bajo el sistema de subcontratación, y de grandes empresas de producción estandarizada, filiales de grandes grupos empresariales con sede en España o en el extranjero, dependientes de distribuidoras europeas.

Cuadro 4. Cooperación Interempresarial (\% del número de relaciones).

\begin{tabular}{|lc|}
\hline Clasificación sectorial & $\%$ \\
\hline GRADO DE TECNOLOGLA & \\
\hline Primario de Baja Tecnología (PBT) & 6,25 \\
Manufacturas de Baja Tecnología (MBT) & 6,25 \\
Manufacturas de Media Tecnologia (MMT) & 18,77 \\
Manufacturas de Media-alta y Alta Tecnología (MMAT) & 18,66 \\
Servicios de Media-alta y Alta Tecnología (SMAT) & 50,01 \\
TOTAL & 100 \\
\hline PATRONES DE INNOvACIÓN & 6,25 \\
\hline Primario Dominado por los Proveedores (PDP) & 10,42 \\
Manufacturas Dominadas por Proveedores (MDP) & 12,43 \\
Manufacturas de Producción a Gran Escala (MPGE) & 8,33 \\
Manufacturas de Proveedores Especializados (MPE) & 12,5 \\
Manufacturas de Base Científica (MBC) & 50,01 \\
Servicios de Base Científica y de Proveedores Especializados (SBC/SPE) & 100 \\
\hline TOTAL & \\
\hline
\end{tabular}

Elaboración propia a partir de las encuestas. 
Cuadro 5. Intensidad Tecnológica y Patrones de Innovación de las empresas cooperantes (\% número de relaciones con respecto al total de los sectores).

\begin{tabular}{|c|c|c|c|c|c|c|c|c|c|}
\hline EMPRESA DE I+D & & & EMI & $\mathbf{R}$ & $\mathbf{P}$ & EAN & & & \\
\hline CLASIFICACIÓN SECTORIAL & PBT & MBT & MMT & MMAT & MAT & SBT & SMAT & SAT & TOTAL \\
\hline GRADO DE TECNOLOGIA & & & & & & & & & \\
\hline Primario de Baja Tecnología (PBT) & 100,0 & 0,0 & 0,0 & 0,0 & 0,0 & 0,0 & 0,0 & 0,0 & 100 \\
\hline ología (MBT) & 0,0 & 0,0 & 0,0 & 66,7 & 0,0 & 0,0 & 33,3 & 0,0 & 100 \\
\hline Manufacturas de Media & 10,0 & 0,0 & 20,0 & 23,3 & 0,0 & 20,0 & 26,7 & 0,0 & 100 \\
\hline y Alta Tecnología (MMAT) & 0,0 & 0,0 & 0,0 & 68,8 & 6,3 & 0,0 & 18,8 & 6,3 & 100 \\
\hline logía (SMT) & 0,0 & 0,0 & 0,0 & 0,0 & 0,0 & 0,0 & 50,0 & 50,0 & 100 \\
\hline Servicios de Media-alta y Alta Tecnología (SAT) & 17,8 & 11,1 & 17,6 & 11,4 & 6,7 & 0,0 & 35,4 & 0,0 & 100 \\
\hline TOTAL & 12,7 & 2,1 & 9,6 & 30,8 & 2,8 & 6,3 & 28,0 & 7,8 & 100 \\
\hline PATR & & & & & & & & & \\
\hline Primar & 100,0 & 0,0 & 0,0 & 0,0 & 0,0 & 0,0 & 0,0 & 0,0 & 100 \\
\hline Manufacturas Dominadas por Proveed & 0,0 & 0,0 & 33,3 & 33,3 & 0,0 & 0,0 & 0,0 & 33,3 & 100 \\
\hline Manufactu & 0,0 & 0,0 & 12,5 & 50,0 & 12,5 & 12,5 & 0,0 & 12,5 & 100 \\
\hline Manufacturas de Proveedores Especializados (MPE) & 0,0 & 0,0 & 33,3 & 13,3 & 6,7 & 0,0 & 20,0 & 26,7 & 100 \\
\hline Manufacturas de Base Cientifica (MBC) & 25,0 & 0,0 & 0,0 & 0,0 & 50,0 & 0,0 & 0,0 & 25,0 & 100 \\
\hline Servicios de Base Cientifica y Proveedores Especi: & 10,7 & 6,7 & 10,6 & 4,9 & 6,0 & 20,0 & 0,0 & 41,2 & 100 \\
\hline TOTAL & 12,7 & 2,1 & 17,4 & 14,0 & 11,8 & 7,8 & 6,3 & 28,0 & 100 \\
\hline
\end{tabular}

Elaboración propia a partir de las encuestas.

\section{Los flujos espaciales de compra de tecnología inmaterial.}

La estrategia innovadora de las empresas no se basa actualmente en la adquisición y explotación de tecnologías complejas (patentes, Know-How, Modelos de Utilidad) (cuadro 6 y 7). La forma de innovar de la mayor parte de ellas viene marcada por el deseo de mejora y reducción de los costes de producción, y para esto adquieren nuevos procesos de fabricación o nuevos métodos de producción (adquisición tecnológica). Aunque para otras se une a la compra de tecnología el desarrollo de actividades de I+D e innovadoras, resultado de la existencia de un mercado potencial en donde la calidad y la diferenciación del producto las hacen competitivas (51,57\% de las empresas) (Ruiz, 2003).

En función de las relaciones que se establecen para la adquisición de tecnología, el proceso innovador de las firmas depende tecnológicamente del mercado foráneo, nacional y extranjero. En la región sólo se adquiere el 25,6\% del total de tecnologías mientras que en España el 41,1 \% y en el extranjero el 33,3\% (Figura 1). La dimensión espacial de la compra varía según los tres grandes sectores de la economía y la disponibilidad de recursos tecnológicos regionales. Las empresas del sector agrario acuden tecnológicamente al mercado español y un poco al mercado extranjero. Este último junto con el nacional suministra los más modernos equipos y tecnologías al sector industrial. Mientras que los servicios avanzados se abastecen principalmente de tecnologías españolas y andaluzas (cuadro 8). 
Cuadro 6. Compra de Tecnología según tipo y origen (\% del número de empresas).

\begin{tabular}{|lccccccccc|}
\hline ORIGEN/FIGURAS & PAT $^{*}$ & M.U & K-H & AS.T & B.EQ & SOFT & MARCAS & N.C. & D. y M. \\
\hline ANDALUCIA & 2,1 & 1,1 & 1,1 & 8,4 & 11,6 & 5,3 & 0,0 & 1,1 & 1,1 \\
ESPAÑA & 1,1 & 0,0 & 8,4 & 13,7 & 23,2 & 14,7 & 2,1 & 2,1 & 1,1 \\
EXTRANJERO & 2,1 & 1,1 & 6,3 & 10,5 & 25,3 & 6,3 & 4,2 & 0,0 & 0,0 \\
TOTAL & 5,3 & 2,1 & 15,8 & 32,6 & 60,0 & 26,3 & 6,3 & 3,2 & 2,1 \\
\hline
\end{tabular}

Elaboración propia a partir de las encuestas.

* PAT: Patentes; M.U.: Modelos de Utilidad; K.-H.: KNOW-HOW; AS. T: Asistencia Técnica; B.EQ: Bienes de Equipo; SOFT: Software; N.C.: Nombre Comercial; D. y M.: Dibujos y Modelos.

Cuadro 7. Procedencia espacial de las tecnologías adquiridas ( $\%$ del número de tecnologías).

\begin{tabular}{|lcccccccccc|}
\hline ORIGEN/FIGURAS & PAT $^{*}$ & M.U & K-H & AS.T & B.EQ & SOFT & MARCAS & N.C. & D. y & M. TOTAL \\
\hline ANDALUCIA & 6,5 & 2,2 & 2,2 & 23,9 & 23,9 & 13,0 & 0,0 & 2,2 & 2,2 & 100 \\
ESPAÑA & 1,4 & 0,0 & 13,5 & 23,0 & 35,1 & 18,9 & 2,7 & 2,7 & 1,4 & 100 \\
EXTRANJERO & 6,7 & 1,7 & 13,3 & 21,7 & 40,0 & 10,0 & 6,7 & 0,0 & 0,0 & 100 \\
TOTAL & 4,4 & $\mathbf{1 , 1}$ & 10,6 & 22,8 & 33,9 & 14,4 & 3,3 & 1,7 & 1,1 & 100 \\
\hline
\end{tabular}

Elaboración propia a partir de las encuestas.

* PAT: Patentes; M.U.: Modelos de Utilidad; K.-H.: KNOW-HOW; AS. T: Asistencia Técnica; B.EQ: Bienes de Equipo; SOFT: Software; N.C.: Nombre Comercial; D. y M.: Dibujos y Modelos.

Cuadro 8. Origen espacial de las Tecnologías adquiridas por Subsistema Empresarial de I+D (\% del número de tecnologías)

\begin{tabular}{|l|c|c|c|c|}
\hline CLASIFICACIÓN SECTORIAL & And. * & Esp. & Extr. & Total \\
\hline GRADO DE TECNOLOGIA & 0 & 75 & 25 & 100 \\
\hline Primario Baja Tecnología (PBT) & 0,0 & 40,0 & 60,0 & 100 \\
Manufactura Baja Tecnología (MBT) & 2,9 & 38,4 & 58,8 & 100 \\
Manufactura Media Tecnología (MMT) & 14,2 & 40,0 & 45,7 & 100 \\
Manufactura Media-alta y Alta Tecnología (MMAT) & 0,0 & 75,0 & 25,0 & 100 \\
Servicio Baja Tecnología (SBT) & 60,9 & 30,5 & 8,5 & 100 \\
Servicio Media Tecnología (SMT) & 26,7 & 41,7 & 31,7 & 100 \\
Servicio Alta Tecnología (SAT) & 20,8 & 41,5 & 37,7 & 100 \\
TOTAL & & & & \\
\hline PATRONES DE INNOvACIÓN & 0,0 & 75,0 & 25,0 & 100 \\
\hline Primario Dominado por los Proveedores (PDP) & 0,0 & 45,0 & 55,0 & 100 \\
Manufacturas Dominadas por los Proveedores (MDP) & 11,9 & 35,5 & 52,6 & 100 \\
Manufacturas de producción a gran escala (MPGE) & 11,8 & 59,2 & 29,0 & 100 \\
Manufacturas de Proveedores Especializados (MPE) & 0,0 & 20,8 & 79,2 & 100 \\
Manufacturas de Base Científica (MBC) & 0,0 & 75,0 & 25,0 & 100 \\
Servicios de Producción a Gran Escala (SPGE) & 46,3 & 35,3 & 18,4 & 100 \\
Servicios de Base Científica y de Proveedores Especializados (SPE/ SBC) & 20,8 & 41,5 & 37,7 & 100 \\
TOTAL & &
\end{tabular}

Elaboración propia a partir de las encuestas.

* And.: Andalucía; Esp.: España; Extr.: extranjero. 
Según los datos aportados por las encuestas, el 89\% de las empresas industriales y algo más de la mitad de la de los servicios avanzados han comprado tecnología en el extranjero. Igualmente es alto el porcentaje de empresas tanto manufactureras como del terciario avanzado que las adquieren en el territorio nacional. Mientras que al ámbito regional recurren mayoritariamente las de servicios avanzados (43\%) y sólo el $20 \%$ de las industriales.

\section{Las relaciones con la administración europea, nacional y autonómica}

Las ayudas públicas a la modernización tecnológica del tejido productivo andaluz (desarrollo de proyectos de $\mathrm{I}+\mathrm{D}$, compra de bienes de equipo, adquisición de servicios avanzados, implantación de sistemas de calidad, etc.) han sido importantes para más de la mitad de las empresas estudiadas (57\%) como se puede apreciar en la Figura 1, y está claramente correlacionada con la cooperación con centros públicos de $I+D$ y con la adquisición de tecnología material. En función del sector económico y de su intensidad tecnológica se aprecia (cuadro 9 y 10):

a) La importancia de la financiación pública para las empresas agrarias.

b) Como las manufacturas clasificadas teóricamente como de mayor complejidad tecnológica acuden a la administración para la modernización tecnológica de sus instalaciones y la introducción de sistemas de calidad. Los sectores agroalimentario, minerales no metálicos, material de transporte e Ingeniería reciben ayudas públicas tanto para proyectos de I+D como de modernización. Y otros como el metalúrgico y el eléctrico-electrónico principalmente se acogen a la línea de modernización tecnológica .

c) La poca relevancia de la financiación pública en el desarrollo de las actividades de I+D y modernización tecnológica en las empresas de servicios avanzados.

Cuadro 9. Financiación pública por intensidad tecnológica del Subsistema Empresarial de I+D.

(\% del número de empresas)

\begin{tabular}{|lc|}
\hline INTENSIDAD TECNOLÓGICA & EMPRESAS \\
\hline Primario de Baja Tecnología (PBT) & 100,0 \\
Manufacturas de Baja Tecnología (MBT) & 100,0 \\
Manufacturas de Media Tecnología (MMT) & 87,1 \\
Manufacturas de Media-alta y Alta Tecnología (MMAT) & 69,3 \\
Servicios de Baja Tecnología (SBT) & 100,0 \\
Servicios de Media Tecnología (SMT) & 4,5 \\
Servicios de Alta Tecnología (SAT) & 16,7 \\
TOTAL & 56,9 \\
\hline
\end{tabular}

Elaboración propia a partir de las encuestas. 
Cuadro 10: Tipología de proyectos acogidos a la Financiación Pública.

(\% del número de empresas sobre el total del sector)

\begin{tabular}{|lccc|}
\hline INTENSIDAD TECNOLÓGICA & I+D & *MOD. & CAL. \\
\hline Primario de Baja Tecnología (PBT) & 100,0 & 0,0 & 0,0 \\
Manufacturas de Baja Tecnología (MBT) & 66,7 & 33,3 & 0,0 \\
Manufacturas de Media Tecnología (MMT) & 53,3 & 60,0 & 23,3 \\
Manufacturas de Media-alta y Alta Tecnologia (MMAT) & 66,7 & 60,4 & 4,2 \\
Servicios de Media Tecnología (SMT) & 25,0 & 25,0 & 0,0 \\
Servicios de Alta Tecnología (SAT) & 33,3 & 0,0 & 0,0 \\
TOTAL & 47,4 & 35,5 & 7,0 \\
\hline
\end{tabular}

Elaboración propia a partir de las encuestas.

*MOD.: Modernización.

CAL.: Calidad.

Las fronteras geográficas o el ámbito territorial al que acceden las firmas para subvencionar el desarrollo de actividades de I+D (Figura 1 y cuadros 11 y 12) viene condicionado por varios factores que se pueden simplificar en el tamaño, capital de control y grado tecnológico de las empresas. Así:

a) La mayor parte de los fondos públicos destinados a la I+D regional procede de la administración nacional a través de contratos de investigación asociados al Plan Nacional de Investigación Científica y Desarrollo Tecnológico. Pero los recursos estatales, $81 \%$ de la financiación pública, se concentran en el 16,8\% de las empresas encuestadas ( $29 \%$ de las que se acogen a ayudas públicas). Se trata tanto de firmas de grandes dimensiones y participadas por capital nacional pertenecientes a la industria manufacturera tradicional de baja tecnología (cuero y calzado) como de grado medio (productos minerales no metálicos, papel y edición, maquinaria y equipo mecánico) y el sector de la construcción.

b) La Comunidad Autónoma apenas aporta recursos ( $18 \%$ del total) a la I+D privada pero apoya a un mayor número de firmas (31,6\% de las empresas de la muestra y $54,57 \%$ de las asistidas) a través de subvenciones. Especialmente PYMEs regionales, industriales tanto de baja como de media y alta tecnología y del subsector agrario.

c) Mientras que los fondos extranjeros públicos, gestionados por la administración central, son poco cuantiosos y principalmente provienen del Programa Marco de I+D de la Unión Europea. Las empresas que acceden a proyectos europeos sólo representan el 9,5\% de las empresas encuestadas y el 16,40\% de las ayudadas. Se trata de empresas pertenecientes a ramas industriales de media y media-alta y alta tecnología con fuerte presencia en la economía regional y participadas por capital foráneo: agroalimentario, químico y, en menor medida, maquinaria y equipo mecánico. 
Cuadro 11. Origen de la Financiación Pública de la $1+D(\%$ del gasto en I+D).

\begin{tabular}{|l|c|c|c|c|c|c|}
\hline GRADO TECNOLÓGICO & $\begin{array}{c}\text { Fondos } \\
\text { propios }\end{array}$ & $\begin{array}{c}\text { Fondos otras } \\
\text { empresas } \\
\text { españolas }\end{array}$ & $\begin{array}{c}\text { Financiación } \\
\text { Pública }\end{array}$ & $\begin{array}{c}\text { Otra } \\
\text { Financiación } \\
\text { Nacional }\end{array}$ & $\begin{array}{c}\text { Fondos } \\
\text { Extranjeros }\end{array}$ & Total \\
\hline Primario Baja Tecnología (PBT) & 77,4 & 14,8 & 7,3 & 0,0 & 0,7 & 100 \\
Manufactura Baja Tecnología (MBT) & 72,4 & 0,0 & 23,0 & 0,0 & 4,7 & 100 \\
Manufactura Media Tecnología (MMT) & 70,3 & 3,2 & 12,0 & 0,0 & 14,5 & 100 \\
Manufacturas Media-alta y Alta Tecnología (MMAT) & 63,3 & 8,8 & 12,7 & 0,0 & 15,1 & 100 \\
Manufacturas Alta Tecnología (MAT) & 89,3 & 0,0 & 2,1 & 0,0 & 8,6 & 100 \\
Servicios Baja Tecnología (SBT) & 73,7 & 0,3 & 19,5 & 0,0 & 6,5 & 100 \\
Servicios Media-alta y Alta Tecnología (SMAT) & 79,6 & 3,0 & 13,6 & 0,0 & 3,8 & 100 \\
TOTAL & 72,4 & 4,4 & 13,7 & 0,0 & 9,4 & 100 \\
\hline
\end{tabular}

Elaboración propia a partir de las encuestas.

Cuadro 12. Origen espacial de la Financiación pública de la l+D ( $\%$ del gasto en l+D).

\begin{tabular}{|l|c|c|c|c|c|c|c|}
\hline \multirow{2}{*}{ GRADO TECNOLÓGICo } & \multicolumn{2}{|c|}{$\begin{array}{c}\text { *ADM. } \\
\text { CENTRAL }\end{array}$} & \multicolumn{2}{c|}{$\begin{array}{c}\text { ADM. } \\
\text { AUTONÓMICA }\end{array}$} & \multicolumn{2}{c|}{$\begin{array}{c}\text { ADM. } \\
\text { LOCAL }\end{array}$} & TOTAL \\
\cline { 2 - 7 } & Subv & Contr & Subv & Contr & Subv & Contr & \\
\hline Primario Baja Tecnología (PBT) & 28,8 & 0,0 & 35,2 & 36,0 & 0,0 & 0,0 & 100 \\
Manufacturas Baja Tecnología (MBT) & 33,5 & 0,0 & 66,5 & 0,0 & 0,0 & 0,0 & 100 \\
Manufacturas Media Tecnología (MMT) & 69,4 & 22,7 & 4,2 & 2,2 & 1,4 & 0,0 & 100 \\
Manufacturas Media-alta y Alta Tecnología (MMAT) & 60,7 & 36,9 & 2,4 & 0,0 & 0,0 & 0,0 & 100 \\
Manufacturas Alta Tecnología (MAT) & 91,6 & 0,0 & 8,4 & 0,0 & 0,0 & 0,0 & 100 \\
Servicios Baja Tecnología (SBT) & 44,3 & 50,0 & 5,7 & 0,0 & 0,0 & 0,0 & 100 \\
Servicios Media-alta y Alta Tecnología (SMAT) & 49,1 & 11,5 & 39,4 & 0,0 & 0,0 & 0,0 & 100 \\
Total & 56,8 & 24,2 & 15,1 & 3,5 & 0,4 & 0,0 & 100 \\
\hline
\end{tabular}

Elaboración propia a partir de las encuestas.

* ADM: Administración; Subv: Subvención; Contr.: Contrato.

\section{Los vínculos de mercado y sus fronteras geográficas}

Ante un lánguido sistema de cooperación y de compra de tecnología inmaterial, el desarrollo de actividades de $\mathrm{I}+\mathrm{D}$ del sector empresarial andaluz está ligado a las relaciones de producción, pero no sólo a la compra de bienes de equipo que mejoren y reducen los costes de producción sino también a toda aquella tecnología que fluye en las relaciones proveedor- cliente (inputs-outputs). De tal forma que para el 55,2 \% de las empresas la fuente de conocimiento para la realización de I+D está en las actividades de producción de los clientes y proveedores, especialmente en los primeros (Figura 1).

Las relaciones con clientes son fundamentales para las empresas de servicios (comercio, informática, consultoras, ingeniería, etc.) y las manufacturas de media-alta y alta tecnología (químico, caucho y materias plásticas, maquinaria y electrónico). En tanto que los proveedores sólo son importantes en el sector agroalimentario y unido a los clientes para el papel, material de transporte e Internet (cuadro 13). 
El análisis conjunto del ámbito espacial de compra-venta y el sectorial muestra como las relaciones con clientes tienen diferentes dimensiones espaciales en función del sector económico:

a) Los clientes regionales sólo juegan un gran papel en la I+D de las empresas de servicios tanto de baja tecnología como de media-alta y alta tecnología. En particular para los servicios energéticos, gas y agua la $\mathrm{I}+\mathrm{D}$ viene impulsada por todas las ramas de la economía andaluza. Mientras que en los servicios avanzados es inducida por la demanda de la industria de media tecnología regional, en especial por el sector agroalimentario ( $62,96 \%$ de los contratos con la industria).

b) Y los clientes foráneos, principalmente nacionales, de baja tecnología (servicios comerciales y manufacturas) para las empresas de los sectores de media-alta y alta tecnología de base científica y de proveedores especializados (químico, caucho y materias plásticas, maquinaria y del sector eléctrico-electrónico).

Por el contrario, las relaciones cliente-proveedor son importantes para las industrias dominadas por proveedores (papel, edición, impresión y reproducción) y productores a gran escala como el material de transporte. Se trata de proveedores principalmente foráneos tanto de baja, media o alta tecnología, y de clientes de media y baja tecnología (agroalimentario y comercial agrario) regionales para el primero y foráneos para el segundo. Mientras que sólo las empresas del sector agroalimentario encuentran su fuente de ideas, conocimiento, en los proveedores regionales del propio sector (media tecnología) y de baja en el agrario.

Cuadro 12. Peso del mercado como fuente de conocimiento para las empresas de $1+D$. (\% del número de empresas con respecto al total del sector).

\begin{tabular}{|l|c|c|c|}
\hline CLASIFICACIÓN SECTORIAL & CLIENTES & *PROV. & TOTAL \\
\hline INTENSIDAD TECNOLÓGICA & 0 & 0 & 0 \\
\hline Primario Baja Tecnología (PB') & 0 & 0 & 0 \\
Manufactura Baja Tecnología (MBT) & 38,10 & 47,62 & 52,38 \\
Manufactura Media Tecnología (MMT) & 57,26 & 15,00 & 65,60 \\
Manufactura Media-alta y Alta Tecnología (MMAT) & 100,00 & 0,00 & 100,00 \\
Servicio Baja Tecnología (SBT) & 66,44 & 18,64 & 66,33 \\
Servicio Media-alta y Alta Tecnología (SMAT) & 50,82 & 18,34 & 55,23 \\
TOTAL & & & \\
\hline PATRÓNES DE INNOVACIóN & 0,00 & 0,00 & 0,00 \\
\hline Primario Dominado por los Proveedores (PDP) & 50,00 & 50,00 & 50,00 \\
Manufacturas Dominadas por los Proveedores (MDP) & 9,82 & 16,96 & 26,79 \\
Manufacturas de Producción a Gran Escala (MPGE) & 47,32 & 0,00 & 47,32 \\
Manufacturas de Proveedores Especializados (MPE) & 83,33 & 25,00 & 91,67 \\
Manufacturas de Base Científica (MBC) & 66,44 & 18,64 & 66,33 \\
Servicios de Producción a Gran Escala (SPGE) & 100,00 & 0,00 & 100,00 \\
Servicios de Base Científica y de proveedores Especializados (SBC/SPE) & 50,82 & 18,34 & 55,23 \\
TOTAL & &
\end{tabular}

Elaboración propia a partir de las encuestas.

*PROV.: Proveedores. 


\section{Proceso innovador y difusión territorial de la innovación}

A modo de conclusión los flujos tecnológicos que se desarrollan entre el sector empresas (I+D) y otros agentes del sistema de innovación regional son de escasa magnitud. No obstante las relaciones entre éstos juegan un gran papel en el proceso innovador de las empresas estudiadas, diferenciado en función de la intensidad tecnológica que:

a) Para las empresas agrarias la realización de actividades de I+D está unida a la cooperación con Opis y la Universidad regional, a las subvenciones de la Administración autonómica y a la subcontratación local.

b) Las manufacturas de baja tecnología efectúan actividades de I+D subvencionadas por la administración autonómica en cooperación con las Opis y Universidades regionales y nacionales; y con otras empresas europeas proveedoras de insumos industriales y de servicios de media-alta tecnología.

c) Las de media tecnología realizan proyectos de I+D en cooperación con Opis regionales financiados por fondos extranjeros y de la administración central. Con una propensión a cooperar con otras empresas comparable al resto de sectores, es especialmente significativa la diversidad sectorial de las empresas cooperantes. Las actividades de $\mathrm{I}+\mathrm{D}$ se han acompañado de una renovación de las instalaciones y equipo productivo al amparo de las políticas de modernización tecnológica autonómica y nacional.

d) Y para las de media-alta y alta tecnología, la I+D está estrechamente vinculada: 1) a las relaciones de cooperación con empresas del propio sector y servicios de media-alta y alta tecnología en el ámbito regional; y 2) al contacto con clientes foráneos. Unas actividades que se desarrollan en el marco de programas de I+D nacionales y con financiación extranjera. Para ello las firmas no sólo han implementado toda una estrategia organizativa sino que también han llevado a cabo un proceso de modernización tecnológica de la producción al abrigo de ayudas públicas.

Por otra parte, el análisis de las relaciones/redes espaciales de las empresas con el subsistema científico muestra la debilidad del sistema ciencia-empresarial andaluz, no sólo por el escaso número de empresas de I+D que están inmersas en él sino también porque el ámbito regional sólo es importante para las empresas agrarias, las manufacturas de media tecnología y los servicios de baja-media tecnología. Sin embargo los sectores de mayor complejidad tecnológica tienen suficiente capacidad de acceso al ámbito nacional, aunque en muchos casos viene motivada por la falta de oferta regional. Así las firmas de media-alta y alta tecnología contabilizan el $69,2 \%$ de las relaciones que se establecen con centros nacionales. 
Las fronteras espaciales de la red quedan definidas por factores territoriales y sectoriales:

- Las relaciones entre el subsistema empresarial y el científico de I+D regional tiene dos claras componentes, una territorial y otra sectorial. Por una parte se asocia a la proximidad geográfica y por otra al grado tecnológico de las ramas. En particular está vinculada a sectores punteros como el eléctrico-electrónico y material de transporte, a ramas con gran peso en la economía y en la I+D andaluza como el agroalimentario, o a sectores muy apoyados por la administración andaluza (metalúrgico).

- En cuanto al área espacial de adquisición de tecnología, el sistema de innovación regional abastece principalmente a los servicios avanzados, particularmente de ingeniería. La industria manufacturera de I+D no encuentra las tecnologías necesarias o más modernas en el mercado regional. Sólo algunas industrias de media y media-alta tecnología (agroalimentaria, maquinaria y material de transporte) compran hasta cierto punto en Andalucía. La empresa industrial recurre mayoritariamente a proveedores de equipos y servicios avanzados extranjeros, si bien algunos de ellos son canalizados a través de intermediarios nacionales. Al tiempo que los propios servicios avanzados, con fuerte presencia en la región, también acuden al Know-How y software nacional.

La I+D regional del sector industrial y de servicios de baja tecnología está asociada en los últimos cinco años a la incorporación de nuevos y avanzados equipos de producción. Una renovación tecnológica que ha permitido la diferenciación y el aumento de la calidad de los productos; y que consiste sobre todo en la introducción de sistemas de organización flexible en el sector agroalimentario y del papel, fabricación asistida por ordenador en el papel, metalúrgico, químico y caucho-materias plásticas y sistemas de Diseño por ordenador CAD-CAM en el electrónico y maquinaria. Pero por otra parte supone una dependencia tecnológica de proveedores de equipo y de inputs especializados foráneos puesto que la región sólo despunta en el suministro de ingeniería a la industria de media tecnología regional.

- Si el análisis se extiende a todas las relaciones de mercado se aprecia como para la industria regional a mayor grado tecnológico mayor dependencia de fuentes geográficamente externas y de demandas poco exigentes. Factor que explica una vez más el rango o valor del proceso y producto de la innovación en las manufacturas de media-alta tecnología andaluza, básicamente una I+D de desarrollo o investigación aplicada a la mejora de productos. Por el contrario la I+D de los servicios de alta tecnología está más integrada con las necesidades del tejido productivo andaluz, en concreto la ingeniería y consultoría con las firmas de media tecnología. Pese a todo, los servicios de producción y 
de información no se adecuan a la demanda de las manufacturas que desarrollan actividades de $\mathrm{I}+\mathrm{D}$ en Andalucía.

- Además casi la mitad de las empresas estudiadas han accedido a las ayudas públicas para financiar las actividades de I+D. Especialmente lo hace la industria, y sobre todo las firmas pertenecientes a ramas tradicionales como el papel, metalúrgico, otras industrias manufactureras y de media tecnología como el Caucho y Materias plásticas con fuerte presencia de la PYME. Ahora bien, la homogeneidad de criterios entre las distintas administraciones en la política científica y la fuerte concentración de los recursos en un número muy reducido de empresas de gran tamaño está produciendo un aumento de las diferencias entre la PYME y la gran empresa industrial en términos de innovación. Los principales recursos emanan de un entorno foráneo, principalmente nacional, en el marco de programas con alto grado de exigencia en el ámbito de la innovación y la solvencia empresarial, y en la mayor parte de los casos inaccesibles para la PYME andaluza. Frente a esto, la administración autonómica pretende que su política sea más equitativa sectorialmente pero no dispone de los suficientes recursos.

No obstante el sistema regional de innovación se está formando gracias, entre otros motivos, al requisito que prevalece en todas las convocatorias de cooperar con otras empresas y/o centros. Y que para el caso andaluz ha favorecido principalmente las relaciones con el subsistema científico regional. El $41 \%$ de las empresas que han recibido ayudas han cooperado con otras instituciones y empresas en el desarrollo de proyectos de I+D. La mayor parte de las redes de cooperación sobrepasan las fronteras regionales, con un peso importante de los centros nacionales y empresas foráneas participantes, excepto para las de servicios avanzados de Consultoría e Internet.

Por todo ello, los procesos de difusión de la innovación a escala regional y local, a través de flujos formales e informales de conocimiento, emanan de las relaciones/redes que configuran las empresas de servicios avanzados y las manufacturas de media tecnología, y desde un punto de vista territorial, de las grandes áreas urbanas de la región. Aunque en la comunidad autónoma existen grandes obstáculos para generar procesos de difusión espacial de la innovación. La carencia de un mínimo subsistema empresarial de $\mathrm{I}+\mathrm{D}$ que estimule la innovación en el sector privado y la inexistencia de demanda de éste mismo dificulta la propagación directa de actividades de $\mathrm{I}+\mathrm{D}$ empresariales e indirectamente de cualquier otra actividad innovadora.

La falta de integración del sector empresas en el sistema de innovación regional limita la formación de un mercado de tecnología material e inmaterial que cree una sinergia interempresarial. Por una parte los servicios avanzados muy concentrados espacialmente son poco demandados, lo que está generando que la oferta sea limitada. Los requisitos necesarios para la creación de flujos formales o informales de conocimiento 
a partir o con las empresas de I+D sólo parecen darse en las grandes áreas urbanas de la región, pudiéndose llegar a afirmar que el sistema empresarial de I+D regional es igual al sistema territorial de I+D del área metropolitana de Sevilla y en menor medida de otros centros urbano-industriales (capitales provinciales) de la región.

En otras palabras, sólo encontramos la suficiente masa crítica de empresas de I+D e innovadoras y de otros agentes del sistema regional de innovación en el área urbana de Sevilla. Este sistema local ejerce su influencia sobre el resto del territorio pero no de una forma continua en el espacio sino que sólo alcanza los principales centros urbanos regionales (capitales provinciales). Por otra, la mayor dispersión territorial de la industria de media tecnología puede originar sinergias que difundan la innovación de medio-bajo contenido tecnológico en el medio rural.

En definitiva las regiones periféricas, Objetivo I en la Unión Europea, tienen grandes problemas para la creación de un mínimo subsistema empresarial de $\mathrm{I}+\mathrm{D}$, conjunto de elementos interrelacionados, que estimule la innovación en el ámbito empresarial, o viceversa, ante la carencia de un tejido innovador que necesite y que por tanto demande I+D. El sistema se retroalimenta de tal forma que cuando emergen las actividades de I+D adoptan unas características (de desarrollo y adaptación, en sectores maduros, muy dependientes del capital exterior) que impiden el cambio tecnológico de la región en su conjunto y del territorio en particular.

\section{Notas}

1 La Comunidad Autónoma de Andalucía es Región Objetivo 1. Según datos aportados por la Comisión Europea (2004) la renta per cápita (SPA) se sitúa en el 63,1 \% de la media de la Unión Europea de los 15 y en el $69,2 \%$ con respecto a la media de la U.E. ampliada a 25 países para el año 2001 . Además mantiene una tasa de paro muy elevada en el 2002 (19,6\%).

2 Para más información sobre la carencia de datos regionales sobre $\mathrm{I}+\mathrm{D}+\mathrm{i}$ veáse la publicación "Las Estadísticas sobre Actividades de Investigación científica y Desarrollo Tecnológico (I+D) en Anclalucía" (Jordá, R. et al., 2002).

3 La Base de clatos de empresas innovadoras de Andalucía incluye todas las empresas-establecimientos que realizan actividades $I+D$ y otras actividades innovadoras en Andalucía. Esta base de datos se elaboró en el año 2001 por el Grupo de Investigación Estudios Geográficos Andaluces en el marco del "Proyecto técnico de diseño metodológico sobre la estadística de Innovación de las empresas e I+D en Andalucía" bajo la dirección de Rosa $M^{2}$ Jordá Borrell; y que recientemente, en el último trimestre del año 2003, ha sido actualizada.

4 A partir del mencionado Directorio de empresas-establecimientos se ha seleccionado una muestra de 164 unidades entre la que realizan actividades de $\mathrm{I}+\mathrm{D}(300)$ por muestreo aleatorio simple. El Criterio de selección ha sido la correspondencia de la distribución sectorial (CNAE) y la localización geogräfica de estas empresas en Andalucía (provincia y comarcas). Para una mayor presencia de los sectores en algu- 
nas comarcas se aplica las normas de redondeo, obteniéndose un número total de 184 encuestas de las que se contactaron efectivamente con 174 y fueron válidos los cuestionarios de 156 . Por lo tanto nos ajustamos al tamaño de la muestra representativa (164) en un $95,12 \%$.

La fórmula aplicada ha sido:

$$
\mathrm{n}=\frac{4 \mathrm{~N}^{*} \mathrm{~V}^{\prime}}{\mathrm{NK} 2+4 \mathrm{~V}^{\prime}}
$$

N: Población total de empresas-establecimientos que realizan actividades de I+D.

$V^{\prime}$ : Cuasivarianza, cuyo cálculo se ha obtenido a partir de la variable gastos en $\mathrm{I}+\mathrm{D}$ de una encuesta piloto realizada a 20 unidades escogidas aleatoriamente del DEA-I+D.

K: Error Muestral. En este caso se ha asumido un error del 10\% de la media ( 84141,7 ) teniendo en cuenta la elevada desviación que muestran los datos de inversión en I+D y el tamaño reducido de la población

5 Es un concepto que parte de la fusión de dos enfoques teóricos. por un lado se basa en los noción de distrito industrial (Marshall, 1919), en lo polos de crecimiento (Perroux, 1955) y en la teoría de los clusters (Porter, 1990). Y por otro se fundamenta en los resultados de la teoría del crecimiento que subrayan la importancia de la innovación en el progreso y desarrollo económico para las áreas geográficas. Las actividades innovadoras generan externalidades positivas (Romer, 1986, 1990; Lucas, 1988) que pueden ser aprovechadas sobre todo por los agentes regionales (Stern, Porter, Furman, 2000). Una definición teórica del concepto de SNRI tiene en cuenta tres aspectos: a. Se trata de un "sistema" en doncie actúan e interactúan distintos agentes e instituciones; b. las actividades analizadas están ligadas a la "innovación"; y c. implica un "enfoque geográfico" (Heijs, 2001).

6 Las Actividades de Investigación y Desarrollo Tecnológico (I+D) quedan definidas como "el conjunto de trabajos creativos que se emprenden de modo sistemático a fin de aumentar el volumen de conocimientos, incluidos el conocimiento del hombre, la cultura y la sociedad, así como la utilización de esa suma de conocimientos para concebir nuevas aplicaciones" (OCDE, 1994).

Las actividades de $\mathrm{I}+\mathrm{D}$ engloban la investigación básica, la investigación aplicada y las actividades de desarrollo tecnológico. Aunque en algunos casos es difícil clistinguir la I+D de otras actividades científico-tecnológicas, será la existencia en el seno de la $\mathrm{I}+\mathrm{D}$ de "un elemento apreciable de creatividad y la resolución de una incertidumbre científica y/o tecnológica; o dicho de otra manera, cuando la solución de un problema no parezca evidente a cualquiera que esté al corriente del conjunto de conocimientos y técnicas básicas utilizadas comúnmente en el sector considerado".

En Otras actividades innovadoras se incluye las actividades innovadoras de carácter tecnológico (nuevos productos introducidas en el mercado y procesos, así como las modificaciones tecnológicas importantes de los mismos) en el mercado (innovación de producto) o utilizada en un proceso de producción (innovación de proceso). Según el Manual de Oslo se pueden resumir en adquisición de tecnología inmaterial (patentes e invenciones no patentadas, licencias, informes de know-how, marcas de fábrica, diseños, modelos de utilidad, compra de servicios de I $+\mathrm{D}$ y otros servicios con un contenido tecnológico; adquisición de maquinaria y equipo; Actividades de diseño e ingeniería industrial, utillaje y lanzamienio de la fabricación; Conjunto de actividades asociadas al lanzamiento de un nuevo producto y Actividades de formación relacionados con la introducción de nuevos productos y procesos.

Por innovaciones no tecnológicas se entiende todos aquellos cambios introducidos por la empresa con el fin de mejorar su funcionamiento y competitividad. Se consideran los siguientes tipos cle novedades: Innovación organizativa; Innovación estratégica; Innovación comercial; Innovación operativa $e$ Innovación "tecnológica" entendiendo por ésta última la adquisición de nuevo equipo no relacionado con nuevos productos o procesos (automatización de procesos rutinarios, eficiencia en los procesos avanzados de información y comunicación, etc.). 
7 Desde la literatura del cambio tecnológico se señala la importancia que tiene la variable sectorial o el área tecnológica en el modo en que tienen lugar los procesos de innovación y difusión, en tanto es la pertenencia a un sector o área tecnológica la que fija el límite espacial del sistema de innovación, y no un límite geográfico determinado (Carlsson, 1995; Bresci y Malerba, 1997). Éstos últimos definen los sistemas sectoriales de innovación (SSI) como el sistema o grupo de empresas activas en el clesarrollo y fabricación de los productos de un sector, y en la generación y utilización de la tecnología de dicho sector. Es decir un SSI está compuesto por las empresas que son activas en las actividades innovadoras de un sector. Estas empresas se relacionan de dos modos: por procesos de competencia y selección en actividades innovadoras y mercantiles. La noción de SSI guarda relación tanto con la taxonomía de Pavitt (1984) que presta atención a las diferencias intersectoriales en el modo de aprendizaje y de protección de la innovación, como con la idea de trayectoria y paradigma tecnológico de Dosi (1988), debido al énfasis en el papel que juegan factores específicos de los sectores productivos y de las propias tecnologías. Estos dos últimos trabajos encuadrados en la teoría de la innovación han supuesto la posibilidad de ampliar el estudio del entorno de las actividades de innovación más allá del mercado, profundizando en el papel que desempeñan las instituciones públicas en la creación de infraestructuras y de servicios avanzados para fomentar la transferencia y difusión de conocimientos nuevos y económicos útiles (Jordá, 1997).

\section{Bibliografía}

Aydalot, PH. (1986 a): Les technologies nouvelles et les formes actuellles de la Division Spatiale du Travail. Dossier $d u$ Centre Economie-Espace-Environnoment, 47. Université de Paris I.

Aydalot, PH. (1986 b): Milieux innovateurs en Europe. París. GREMI.

Bresci, S. and Malerba, F. (1997): Sectorial Innovation Systems: Technological Regimes, Schumpeterian Dynamics, and Spatial Boundaries, en Edquist, C. (ed.) Systems of Innovation. Tecbnologies, Institutions and Organizations. London and Washington, Pinter, p. $130-155$.

Cano, G. (dir.) (2002): Las Comarcas Andaluzas. Sevilla. Ediciones Tartessos.

Carlsson, B. (ed.) (1995); Tecbnological systems and economic performance: the case of factory automation. Dordrecht, Kluver.
Dematteis, G. (1995): Progetto implicito. Milán. Franco Angelo.

Dosi, G. (1988): Sources, Procedures and Micro Economic Effects of Inovation. Joumal of Econoic Literame XXVi.

Edquist, C. (ed.) (1997): Systems of Innovation. Tecbnologies, Institutions and Organizations. London and Washington. Pinter Publishers.

Heijs, J. (2001): Sistemas Nacionales $y$ Regionales de Innovación politica tecnológica: una aproximación teórica. Documento de Trabajo $n^{\circ} 24$. Octubre de 2001. IAIF (Instituto de Análisis Industrial y Financiero de la Universidad Complutense de Madrid).

Jordá, R. (1997): Formación del sistema ciencia-tecnología-industria, en Vallés (coord.) Economia Andaluza. Sevilla, Algaida editores, p. 619-682. 
Jordá, R., Ruiz, F., y Lucendo, A.L. (2000): $E l$ entorno productivo $y$ su incidencia en el desarrollo del proceso innovador de las empresas andaluzas. Sevilla, Instituto de Estadistica de Andalucía.

Jordá, R. (2003 a): Servicios a empresas (SEMP) y Territorio, en López, E., Miranda, J., Posada, J.C., Navarro, J. y García, A. (eds.). Servicios y Transportes en el Desarrollo Territorial de España. Sevilla. Secretariado de Publicaciones de la Universidad de Sevilla, p. 15-243.

Jordá, R. (2003 b): Proceso innovador y capacidad de creación de espacio de los servicios avanzados (SA) en Andalucía. Geograpbicalia, 43, p. 47-79.

Koschatzky, K. (2000): The regionalisation oof innovation polucy in Germany-theoretical Foundations and recent experience. Arbeitspapiere Unternebmen und Región, 1.

Lucas, R. (1988): On the Mechanics of Development Planning. Journal of Monetary Economics, 22.

Lundvall, B.A. (ed.) (1992): National Systems of Innovation: towards a Theory of Innovation and Interactive Learning. London. Pinter.

Marshall (1919): Industry and Trade. London. McMillan.

Méndez, R. (1997 a): Procesos de innovación tecnológica y reorganización del espacio industrial. VI Jornadas de Geografia Industrial. Nuevas Tecnologias, trabajo $y$ localización industrial. Asociación de Geógrafos Españoles (AGE). Grupo de Geografía Industrial. Granada, p. 158-190.

Méndez, R. (1997 b): Geografía Económica. La lógica espacial del capitalismo global. Barcelona, Ariel.
Méndez, R. (2001): Innovación en la pequeña empresa y redes de cooperación para el desarrollo loca, en Martínez Puche, A. (coord.) El Desarrollo Rural/Local Integrado y el Papel de los Poderes Locales. Universidad de Alicante.

Morgan, K. (1997): The Learning Region: Institutions, Innovation and Regional Renewal. Regional Studies, 31, p. 491-503.

OCDE (1994): Proposed Standard Practice for Surveys of Research and Experimental Development. Paris. Frascati-Manual.

OCDE (1997): Révision des classifications des secteurs et des produits de baute technologie. Doc. OCDE/GD (97) 216.

Pavitt, K. (1984): Sectoral Patterns of Technological Change: Towards a Taxonomy and Theory. Research Policy, 13, p. 343-373.

Perroux (1955): Note sur la notion de pôle de croissance. Économie Appliquée, 7.

Porter, M. (1990): The Comparative Advantage of Nations. New York, Free Press and Macmillan.

Romer, M.P. (1986): Increasing Returns and Long-Run Growth. Journal of Political Economy, 94, p. 1002-1037.

Salom, J., Albertos, J.M., Pitarch, D. y Delios, E. (1999): Sistema Urbano e Innovación Industrial en el País Valenciano. Valencia, Universitat de Valencia.

Salom, J. (2003): Innovación y actores locales en los nuevos espacios económicos: un estado de la cuestión. Boletín de la $A G E$, 36, p. $7-30$.

Soete, L. y Miozzo, M. (1989): Trade and Development in Services. A technological 
Perspective, Working Paper, p. 89-031, MERIT, Maastricht.

Ruiz, F. (2003): Las actividades de I+D en el subsistema empresarial de Innovación Andaluz. El Espacio relacional de las empresas de $I+D$. Sevilla. Consejo Económico y Social de la Junta de Andalucía. Tesis doctoral (en prensa).

Stern, S., Porter, M.E. y Furman, J.L. (2000): The Determinants of National Innovative Capacity. Working paper 7876. National Bureau of Economic Research, INC.
Teece, D. (1987): Profiting from technological innovation research, p. 285-305.

Vallés, J. (coord.) (1997a): Economía Andaluza. Sevilla, Algaida Editores.

Vallés, J. (1997b): La economía española ante los retos europeos. Economía española. Madrid, McGraw-Hill, p. 3-64.

Vázquez, A. (1997): Desarrollo endógeno: mecanismos institucionales y culturales. XXIII Reunión de Estudios Regionales: Globalización, Unión Europea, politicas regionales. Valencia, Revista Valenciana d'Estudis Autonómics, 21 (Extraordinario), p. 71-91. 